\title{
Radiação gama e nitrito de sódio na composição química e textura de mortadelas
}

\author{
Gamma radiation and sodium nitrite on chemical composition and \\ textural properties of mortadellas
}

\author{
Monalisa Pereira Dutra ${ }^{\mathrm{I}}$ Eduardo Mendes Ramos ${ }^{\mathrm{I}}{ }^{*}$ Carolina Naves Aroeira ${ }^{\mathrm{I}}$ \\ Alcinéia de Lemos Souza Ramos ${ }^{\mathrm{II}}$ Mauricio Henriques Louzada Silva ${ }^{\mathrm{II}}$ \\ José Luís ContadoII Marcio Tadeu Pereira ${ }^{\text {IV }}$
}

RESUMO

Um delineamento composto central rotacional (DCCR) foi conduzido para estudar os efeitos simultâneos da adição de nitrito (43,6; 150; 256,4; e 300 ppm) e de doses de radiação gama $(2,9 ; 10 ; 17,1$; e $20 \mathrm{kGy})$ nos atributos de textura e parâmetros fisico-químicos de mortadelas. $\mathrm{O}$ pH, composição química e adesividade das mortadelas não foram afetados por nenhuma das variáveis estudadas, enquanto a dureza e a mastigabilidade foram influenciados pela aplicação da radiação gama. Modelos de regressão significativos $(P<0,05)$ foram estabelecidos para a atividade de água, coesividade e flexibilidade das amostras. Estes modelos indicam que os efeitos da irradiação gama sobre estes parâmetros é dependente do nível de nitrito adicionado, sendo o comportamento diferente para amostras com níveis de adição menores que 150 ppm de nitrito daquelas com níveis de adição maiores.

Palavras-chave: perfil de textura, produto emulsionado,
composição química.

\section{ABSTRACT}

A central composite rotational design (DCCR) was conducted to study simultaneously the effects of nitrite additions (43.6, 150, 256.4 and 300 ppm) and gamma irradiation (2.9, 10, 17.1 and $20 \mathrm{kGy}$ ) in texture attributes and physicochemical parameters of bologna-type sausages. The $\mathrm{pH}$, chemical composition and adhesiveness were not affected by any of the variables studied, while hardness and chewiness were influenced by gamma irradiation. Significant models of regression $(P<0.05)$ were established for water activity, cohesiveness and springiness. These models indicate that the effects of gamma irradiation on these parameters are dependent on the level of nitrite added. The effects of irradiation in sausages with less than 150 ppm nitrite were distinct from samples with higher levels of addition.

Key words: texture profile, emulsion-type product, chemical composition.

\section{INTRODUÇÃO}

Nitritos são aditivos adicionados em produtos cárneos como conservantes, inibindo a multiplicação e produção de toxina do Clostridium botulinum (CAMMACK et al., 1999), além de conferir a cor e sabor característicos de produtos curados e prevenir alterações oriundas da rancidez oxidativa dos lipídios (HONIKEL, 2008; BREWER, 2009). Entretanto, devido aos riscos da sua adição na formação de compostos $\mathrm{N}$-nitrosos, reconhecidamente cancerígenos (HOUSER et al., 2003), é que pesquisas têm sugerido formas alternativas para substituir ou, pelo menos, reduzir a quantidade de nitrito adicionada. Uma alternativa viável é o uso da radiação gama (DUTRA et al., 2011), capaz de induzir a radiólise do nitrito e das N-nitrosaminas (AHN et al., 2002a; AHN et al., 2002b).

A resistência do uso de radiação gama em produtos cárneos, no entanto, é devido à possibilidade de alterações indesejáveis nas suas características

IPrograma de Pós-graduação em Ciência dos Alimentos, Departamento de Ciência dos Alimentos (DCA), Universidade Federal de Lavras (UFLA), Lavras, MG, Brasil.

IIDepartamento de Ciência dos Alimentos (DCA), UFLA, 37200-000, Lavras, MG, Brasil. E-mail: emramos@dca.ufla.br. *Autor para correspondência.

IIIInstituto Federal do Sudeste de Minas Gerais (IFSUDESTE-MG), Rio Pomba, MG, Brasil.

${ }^{\mathrm{IV}}$ Centro de Desenvolvimento da Tecnologia Nuclear (CDTN), Comissão Nacional de Energia Nuclear (CNEN), Belo Horizonte, MG, Brasil. 
de qualidade, como perda de textura, cor, sabor e capacidades emulsionante e de retenção de água, induzidos pelos radicais hidroxílicos formados na radiólise da água (BREWER, 2009). Da mesma forma, a adição de nitrito também pode influenciar na textura do produto final, uma vez que reage com resíduos de aminoácidos, formando ligações cruzadas entre as proteínas (MOHLER \& SCHEERER, 1979).

A textura é um dos principais parâmetros para a avaliação da qualidade (RAMOS \& GOMIDE, 2007), sendo que a aceitação dos consumidores para um determinado alimento é extremamente dependente das suas características estruturais. Entretanto, poucos trabalhos relataram os efeitos da adição de nitrito (DONG et al., 2007) ou da irradiação gama (BYUN et al., 2000) sobre a textura de produtos cárneos cozidos, não sendo encontrado nenhum estudo que relatasse estes efeitos em conjunto.

Dessa forma, objetivou-se avaliar simultaneamente os efeitos da adição de diferentes níveis de nitrito e doses de irradiação gama nos atributos de textura e parâmetros físico-químicos de mortadelas.

\section{MATERIAL E MÉTODOS}

Os efeitos dos níveis de nitrito de sódio (43,6; 150; 256,4; e 300ppm), das doses de radiação gama (2,9; 10; 17,1; e 20kGy) e possível interação foram avaliados por um Delineamento Composto Central Rotacional (DCCR), em fatorial 2x2, com quatro pontos fatoriais, quatro pontos axiais e três pontos centrais, totalizando 11 ensaios (PEREIRA et al., 2011), realizados em ordem aleatória.

A elaboração das mortadelas foi conduzida no Laboratório de Processamento de Carnes e Derivados do DCA/UFLA, seguindo a formulação descrita por DUTRA et al. (2011), sendo adicionadas de diferentes níveis de nitrito de sódio, processadas em cutter KJ-10 (Indústrias Jamar, Tupã, SP, Brasil), embutidas (gomos de $\pm 400 \mathrm{~g}$ ) em tripa artificial de poliamida (65mm diâmetro, Walsroder $\mathrm{K}$ plus) e cozidas por imersão em água até $73^{\circ} \mathrm{C}$ (no ponto frio). Após cozimento, as mortadelas foram resfriadas e estocadas em câmara fria $\left(4^{\circ} \mathrm{C}\right)$ por 24 horas, quando foram acondicionadas em caixas térmicas e submetidas a diferentes doses de radiação, conduzidas no Irradiador Gama GB-127 (IR-214; MDS Nordion, Ottawa, Canadá; fonte de cobalto-60; 5 kGy h${ }^{-1}$ ) do Laboratório de Irradiação Gama do Centro de Desenvolvimento da Tecnologia Nuclear (CDTN/CENEN), em Belo Horizonte, MG. As amostras não irradiadas foram mantidas nas mesmas condições de temperatura e por períodos de tempo similares às irradiadas.
As mortadelas foram analisadas quanto à composição química (teor de água, proteínas, gorduras e cinzas), em triplicata, de acordo com os métodos oficiais da AOAC (2002). Os valores de $\mathrm{pH}$ foram obtidos através da inserção de eletrodo combinado, tipo penetração, em três pontos diferentes do produto e da atividade de água, determinada em aparelho Aqualab CX2 (Decagon Devices Inc., Pullman, Estados Unidos).

A avaliação da textura foi conduzida pelo método de análise de perfil de textura (TPA), utilizando um texturômetro TA.XT2i (Stable Micro Systems, Godalming, Inglaterra). Seis amostras (replicatas), cortadas em cubos de $1,0 \mathrm{~cm}$ de aresta, foram duas vezes comprimidas, a uma velocidade de $180 \mathrm{~mm}$ $\min ^{-1}$, até $50 \%$ de seu tamanho. Não houve tempo de repouso entre os dois ciclos de compressão. A curva de deformação com o tempo foi obtida, sendo gerados seis parâmetros de textura (BOURNE, 1978; RAMOS \& GOMIDE, 2007): fraturabilidade, dureza, coesividade, adesividade, flexibilidade e mastigabilidade.

Para cada variável experimental (nitrito e irradiação), a variância foi decomposta nos componentes linear, quadrático e interação, a fim de avaliar a sua importância relativa e o ajuste da seguinte função polinomial de segunda ordem:

$$
\mathrm{Y}=\beta_{0}+\sum_{i=1}^{2} \beta_{i} X_{i}+\sum_{i=1}^{2} \beta_{i t} X_{i}^{2}+\sum_{i=1}^{2} \sum_{j=1}^{2} \beta_{i j} X_{i} X_{j}
$$

Em que $Y$ é a resposta estimada; $B_{0}, B_{i}$, $B_{i i}$ e $B_{i j}$ representam as constantes e coeficientes de regressão do modelo; e $X_{i}, X_{j}$ e $X_{i} X_{j}$ são os níveis das variáveis independentes linear, quadrática e interação, respectivamente.

A significância dos parâmetros da equação para cada variável resposta foi avaliada pelo teste $\mathrm{F}$, utilizando o programa Statistica ${ }^{\circledR} 5.0$ (StatSoft, Tulsa, Estados Unidos). Para a modelagem, foi utilizado o erro puro, avaliando o ajuste da regressão em nível de 5\% de probabilidade e a significância dos coeficientes de regressão ao nível de $10 \%, 5 \%$ e $1 \%$. Na falta de ajuste do modelo completo para o desenvolvimento dos gráficos, foi feita a análise de regressão $(\mathrm{P}<0,05)$ apenas para os coeficientes significativos.

\section{RESULTADOS E DISCUSSÃO}

Os valores médios da composição química e das características físico-químicas das mortadelas são apresentados na tabela 1 . Os valores de $\mathrm{pH}$ e teores de água, proteínas totais, gordura e cinzas das mortadelas não foram afetados $(\mathrm{P}>0,05)$ pela adição de nitrito ou irradiação, estando os produtos em 
concordância com a legislação brasileira (BRASIL, 2000), que preconiza valores mínimos de $12 \%$ para proteínas e máximo de 30\% para a gordura. Entretanto, o teor médio de água encontrado nas mortadelas ficou pouco acima do estabelecido pela legislação (máximo de 65\%).

Para a atividade de água (Aa), o modelo matemático completo foi significativo (Tabela 2), sendo possível construir uma superfície de resposta em função dos níveis de nitrito e doses de irradiação (Figura 1). Maiores efeitos foram observados para a interação da irradiação com a quantidade de nitrito adicionada. Ambas as variáveis afetaram a Aa possivelmente por interferir, de forma distinta, nas interações proteína-água.

Segundo THAKUR \& SINGH (1994), a água, quando irradiada, pode sofrer radiólise levando à sua ionização e consequente rearranjo eletrônico, o que pode ocasionar a produção de radicais livres e íons como radical hidroxil $(\bullet \mathrm{OH})$, elétrons aquosos $\left(\mathrm{e}_{\mathrm{aq}}^{-}\right)$, hidrogênio livre $(\cdot \mathrm{H})$ e próton hidratado $\left(\mathrm{H}_{3} \mathrm{O}^{+}\right)$. Interações com estes radicais livres podem aumentar a formação de peróxido de hidrogênio $\left(\mathrm{H}_{2} \mathrm{O}_{2}\right)$ e pode reagir com o oxigênio $\left(\mathrm{O}_{2}\right)$ dissolvido para formar radicais hidroperoxido (ROO•) em equilíbrio com radicais superóxido $\left(\cdot \mathrm{O}_{2}^{-}\right)$. Por serem altamente reativos, estes produtos interagem quimicamente entre si ou com moléculas próximas (ácidos graxos, aminoácidos, compostos aromáticos, etc) e, como consequência, novas moléculas podem ser danificadas, passando a disputar elétrons com o meio (STEWART, 2001). Dessa forma, é provável que as moléculas de proteína tenham sido afetadas diretamente pela irradiação ou pela reatividade dos radicais livres formados na radiólise da água, acarretando na alteração da proporção de água nas diversas camadas (água constituição, de hidratação e livre) e consequente aumento da Aa das mortadelas. Este efeito foi mais pronunciado para maiores adições de nitrito, que pode reagir com resíduos de aminoácidos, formando ligações cruzadas entre as proteínas (MOHLER \& SCHEERER, 1979), afetando a interação proteína-água e, consequentemente, a Aa. Já os efeitos da redução na Aa com a irradiação, observados quando os valores de nitrito adicionado estão abaixo de 125-150ppm (Figura 1), são mais difíceis de explicar. É provável que esta redução esteja diretamente relacionada à radiólise da água e, ou, às interações com outros componentes presentes no produto, como a fécula de mandioca. SOKHEY \& HANNA (1993) reportaram que o aumento da dose de irradiação produz um aumento nas quantidades de radicais livres de hidratos de carbono, potencializando a despolimerização da molécula de amido e resultando na redução da viscosidade e aumento da solubilidade em água destas moléculas.

Os valores médios dos parâmetros de textura das mortadelas são apresentados na tabela 2, não tendo sido possível detectar o pico de fratura. Para a adesividade, além do modelo completo $\left(\mathrm{P}=0,7193 ; \mathrm{R}^{2}=0,4478\right)$, nenhum dos coeficientes de regressão foram significativos $(\mathrm{P}>0,10)$, indicando que este parâmetro não foi afetado pelo nitrito e ou pela aplicação da irradiação. Para os demais parâmetros de textura, os modelos matemáticos da dureza, coesividade, flexibilidade e mastigabilidade apresentaram coeficientes significativos.

Apenas o coeficiente de regressão (CR) quadrático da irradiação (Irrad x Irrad) foi significativo para os atributos de dureza $(\mathrm{CR}=1,8117$; $\mathrm{P}<0,10)$ e mastigabilidade $(\mathrm{CR}=6,1199 ; \mathrm{P}<0,10)$ das amostras. Entretanto, da mesma forma que o modelo

Tabela 1 - Parâmetros físico-químicos das mortadelas adicionadas de nitrito e irradiadas (Irrad) de acordo com o arranjo experimental.

\begin{tabular}{|c|c|c|c|c|c|c|c|c|}
\hline Ensaio & Nitrito (ppm) & Irrad (kGy) & Teor de água (\%) & Proteínas (\%) & Gorduras (\%) & Cinzas (\%) & $\mathrm{pH}$ & Aa \\
\hline 1 & 43,6 & 2,9 & 67,74 & 16,81 & 11,93 & 2,97 & 6,48 & 0,973 \\
\hline 2 & 256,4 & 2,9 & 66,49 & 17,81 & 12,22 & 2,88 & 6,34 & 0,972 \\
\hline 3 & 43,6 & 17,1 & 66,62 & 16,43 & 13,18 & 3,07 & 6,21 & 0,963 \\
\hline 4 & 256,4 & 17,1 & 67,50 & 16,40 & 13,50 & 3,07 & 6,32 & 0,978 \\
\hline 5 & 0,0 & 10,0 & 67,76 & 17,57 & 11,49 & 3,03 & 6,28 & 0,960 \\
\hline 6 & 300,0 & 10,0 & 67,44 & 17,21 & 11,58 & 2,96 & 6,25 & 0,965 \\
\hline 7 & 150,0 & 0,0 & 68,00 & 17,74 & 10,12 & 2,97 & 6,32 & 0,969 \\
\hline 8 & 150,0 & 20,0 & 67,59 & 17,48 & 10,35 & 3,03 & 6,16 & 0,963 \\
\hline \multirow[t]{3}{*}{$9-11^{*}$} & 150,0 & 10,0 & 67,41 & 17,55 & 11,50 & 2,96 & 6,19 & 0,969 \\
\hline & & Média & 67,40 & 17,28 & 11,72 & 2,99 & 6,27 & 0,968 \\
\hline & & $\mathrm{DP}$ & 0,54 & 0,51 & 1,07 & 0,07 & 0,09 & 0,005 \\
\hline
\end{tabular}

DP = desvio-padrão; Aa =atividade de água.

* média dos 3 pontos centrais. 
Tabela 2 - Coeficientes de regressão (CR) e erro padrão (EP) para as variáveis codificadas dos modelos matemáticos polinomiais, para os valores de atividade de água, coesividade e flexibilidade das mortadelas.

\begin{tabular}{|c|c|c|c|c|c|c|}
\hline & \multicolumn{2}{|c|}{---------Atividade de água------------ } & \multicolumn{2}{|c|}{---------'Coesividade-------------- } & \multicolumn{2}{|c|}{-----------Flexibilidade (mm)----- } \\
\hline & CR & EP & CR & $\mathrm{EP}$ & CR & $\mathrm{EP}$ \\
\hline Constante & $0,9686 * *$ & 0,0004 & 0,7499 & 0,0059 & 5,2369 & 0,0312 \\
\hline $\mathrm{NO}_{2}$ & $0,0026^{* *}$ & 0,0003 & $-0,0278 * *$ & 0,0036 & 0,0271 & 0,0191 \\
\hline $\mathrm{NO}_{2} \times \mathrm{NO}_{2}$ & $-0,0013^{*}$ & 0,0003 & $-0,0077$ & 0,0043 & $-0,1717^{* *}$ & 0,0229 \\
\hline Irrad & $-0,0016^{* *}$ & 0,0003 & $-0,0333 * *$ & 0,0036 & $0,1456 * *$ & 0,0191 \\
\hline Irrad x Irrad & 0,0002 & 0,0003 & $-0,0095$ & 0,0043 & $-0,0208$ & 0,0229 \\
\hline $\mathrm{NO}_{2}$ x Irrad & $0,0040 * * *$ & 0,0004 & $-0,0519 * * *$ & 0,0051 & $0,3337 * * *$ & 0,0270 \\
\hline $\mathrm{R}^{2}$ & 0,5830 & & 0,5216 & & 0,6225 & \\
\hline \multicolumn{7}{|l|}{ P-valor } \\
\hline Regressão & 0,0193 & & 0,0193 & & 0,0183 & \\
\hline LOF & 0,1620 & & 0,0129 & & 0,0182 & \\
\hline
\end{tabular}

$\mathrm{NO}_{2}=$ nitrito; Irrad = Irradiação; LOF = falta de ajuste.

$* * * \mathrm{P}<0,01 ; * * \mathrm{P}<0,05 ; * \mathrm{P}<0,10$.

completo, o modelo matemático ajustado a partir do componente quadrático não foi significativo tanto para a dureza $\left(\mathrm{P}=0,0761 ; \mathrm{R}^{2}=0,4049\right)$, quanto para a mastigabilidade $\left(\mathrm{P}=0,0875 ; \mathrm{R}^{2}=0,2141\right)$. Alterações na textura de carnes e produtos cárneos pela irradiação são teoricamente possíveis através da alteração das propriedades funcionais das proteínas. Trabalhos que tenham avaliado os efeitos da irradiação, especialmente a aplicação de altas doses (>5kGy), na textura de produtos cárneos são escassos. BYUN et al. (2000) não observaram nenhum efeito significativo na dureza, elasticidade e coesividade de mortadelas elaboradas com matéria-prima cárnea irradiada com 3 e 5kGy. Em produtos fermentados, CABEZA et al. (2009) não observaram nenhuma mudança na dureza de salames irradiados até doses de 3kGy, embora GALÁN et al. (2011) tenham reportado uma redução na dureza com a aplicação de 4kGy.

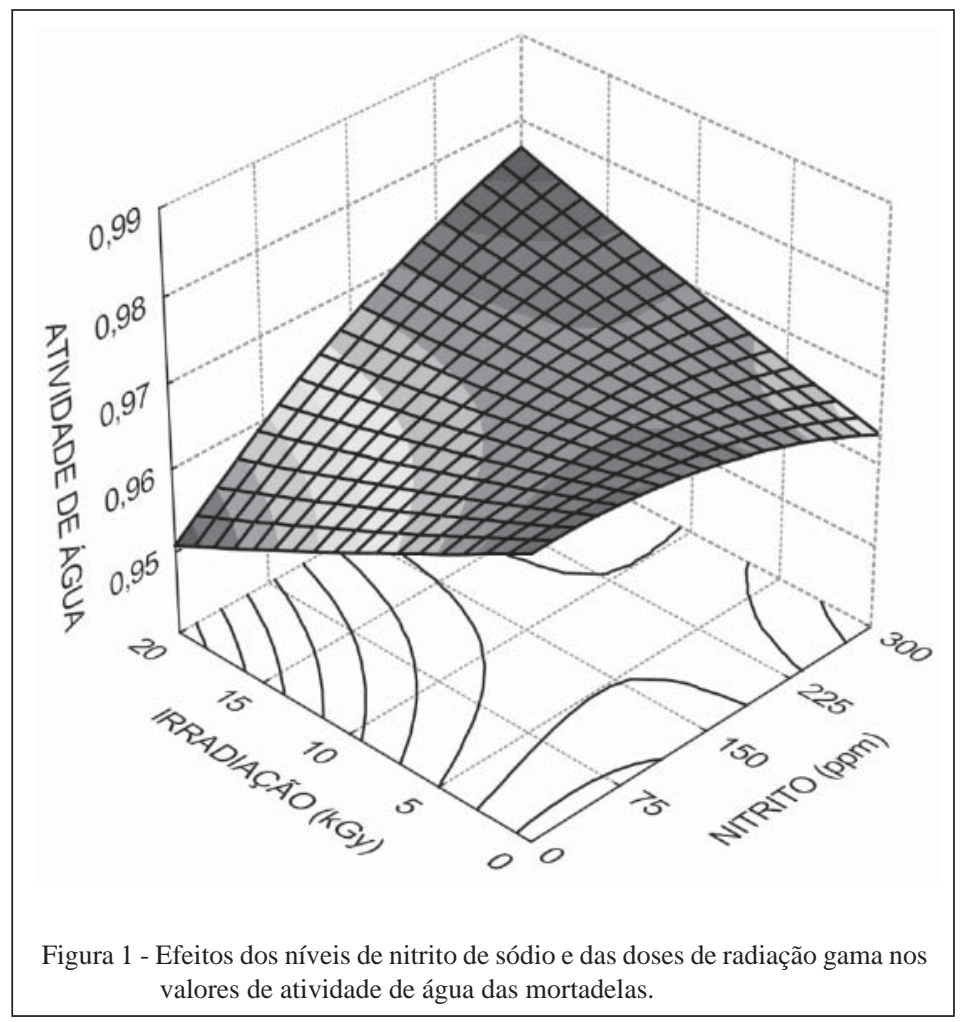

Ciência Rural, v.44, n.6, jun, 2014. 
Embora a mastigabilidade seja um parâmetro secundário dependente da dureza, coesividade e flexibilidade da amostra (BOURNE, 1978), os efeitos significativos observados foram similares ao da dureza, não sendo observado efeito da adição de nitrito. Os parâmetros de coesividade e flexibilidade foram afetados tanto pela irradiação quanto pela quantidade de nitrito adicionado (Tabela 2), sendo os modelos matemáticos representados nas superfícies de resposta da figura 2.

Segundo BOURNE (1978), a coesividade representa a força das ligações internas que determinam a extensão que o alimento é deformado antes da ruptura, podendo ser traduzido

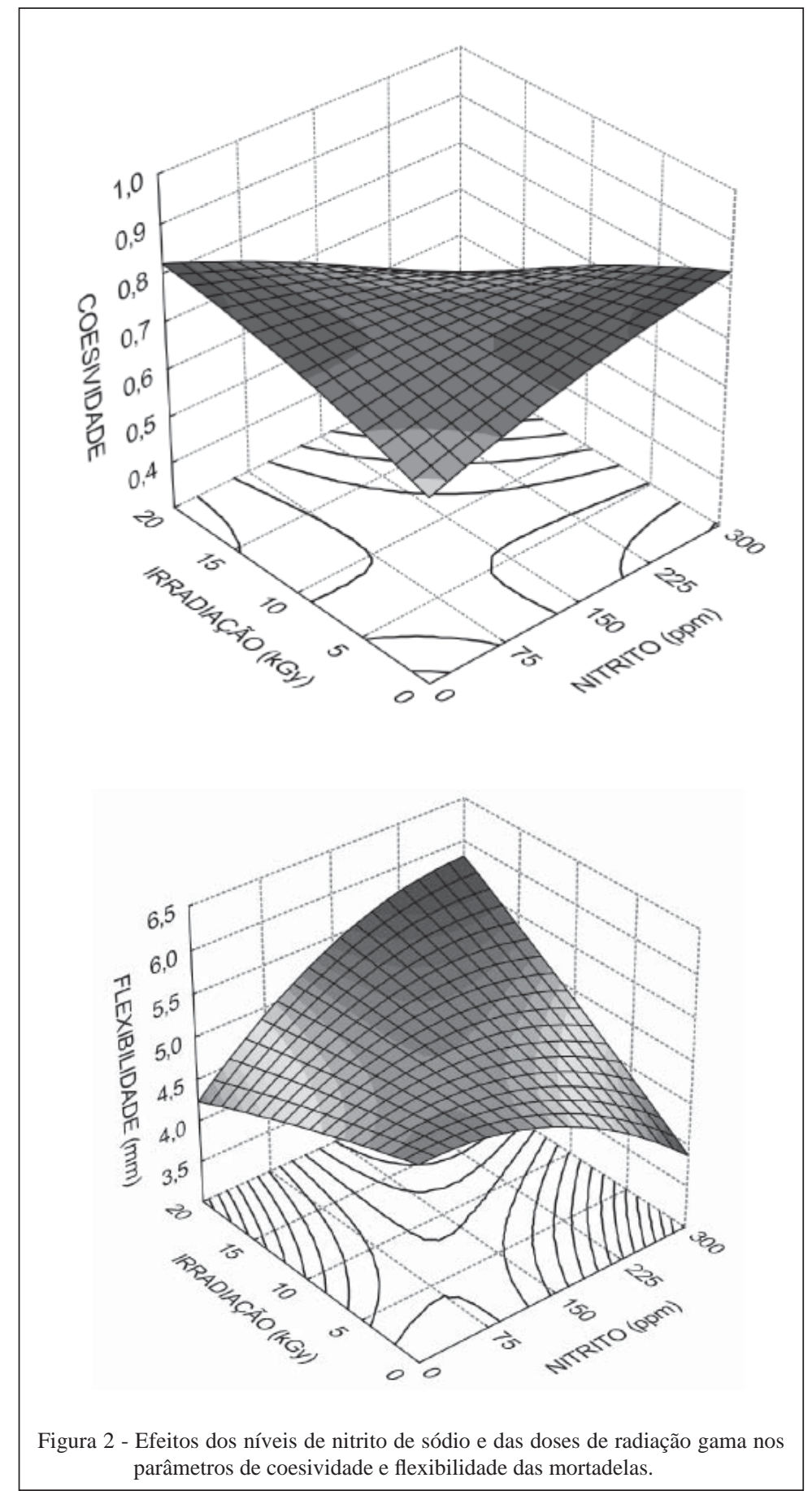

Ciência Rural, v.44, n.6, jun, 2014. 
sensorialmente como mastigável ou consistente, ou seja, a quantidade de deformação sofrida pela amostra quando comprimida pelos dentes molares. Já a flexibilidade, ainda segundo este autor, é a taxa em que o material deformado retorna à sua condição inicial antes da segunda compressão, o que sensorialmente é relacionado à elasticidade da amostra. Ambos os parâmetros são, portanto, importantes na percepção sensorial da textura de produtos cárneos.

Em ambos os parâmetros, a interação entre as variáveis nitrito e irradiação foi significativa, porém o comportamento foi distinto. Em maiores adições de nitrito, há um aumento nos valores de coesividade das amostras, porém, com a aplicação da irradiação, ocorre uma redução nestes valores, quando os níveis de nitrito são inferiores a 150ppm e aumento para níveis de adição maiores. O inverso é observado para o parâmetro flexibilidade (Figura 2), o que leva a concluir que, à medida que a amostra torna-se mais coesa com os tratamentos, menos flexível ela fica.

Conforme anteriormente mencionado, estas alterações na textura são possíveis através da alteração das propriedades gélicas das proteínas e de polissacarídeos, seja por efeitos diretos ou indiretos da radiólise (STEWART, 2001; BREWER, 2009) ou por interações induzidas pelo nitrito. De fato, a textura firme de curados é creditada a interações entre proteínas, o que é teoricamente possível quando se considera a reação do nitrito com grupos sufidrilas (-SH) de resíduos aminoacídicos das proteínas, formando pontes dissulfetos (-S-S-) termicamente estáveis entre as proteínas da carne (MOHLER e SCHEERER, 1979).

Com relação aos efeitos da adição de nitrito na textura dos produtos emulsionados, os resultados aqui observados são diferentes dos reportados por DONG et al. (2007), cuja adição de nitrito de sódio (0, 50, 100 e 150ppm) em salsichas foi correlacionada negativamente com a dureza e adesividade e positivamente com a coesividade e flexibilidade. Entretanto, pela figura 2, observa-se que os efeitos da variável nitrito foram bastante sutis, o que pode explicar a diferença de resultados para os demais trabalhos, especialmente se considerarmos que a escolha da velocidade e do percentual de compressão para a condução do teste de TPA, assim como o tamanho e o formato da amostra, são determinantes para a efetividade do teste. Qualquer contribuição do nitrito e, ou, da irradiação à textura é bastante sutil, sendo detectada instrumentalmente apenas em condições particulares. Isto demonstra a complexidade em se entender as características de textura em produtos cárneos curados.

\section{CONCLUSÃO}

Os valores de $\mathrm{pH}$, teores de água, proteínas totais, gordura e cinzas das mortadelas não foram afetados pela adição de nitrito ou irradiação. Porém, diferentes doses de radiação gama e níveis de adição de nitrito afetam a atividade de água e a textura objetiva de mortadelas, especialmente a sua coesividade e flexibilidade, sendo os efeitos da irradiação dependentes da quantidade de nitrito adicionado.

\section{AGRADECIMENTOS}

Os autores agradecem à Fundação de Amparo à Pesquisa do estado de Minas Gerais (FAPEMIG), pelo apoio financeiro à execução do projeto, e à Coordenação de Aperfeiçoamento de Pessoal de Nível Superior (CAPES), pela bolsa de doutorado conferida ao primeiro.

\section{REFERÊNCIAS}

AHN, H.J. et al. Monitoring of nitrite and N-Nitrosamine levels in irradiated pork sausage. Journal of Food Protection, v.65, n.9, p.1493-1497, 2002a.

AHN, H.J. et al. Reduction of carcinogenic N-nitrosamines and residual nitrite in model system sausage by irradiation. Journal of Food Science, v.67, n.4, p.1370-1373, 2002b. Disponível em: <http://dx.doi.org/10.1111/j.1365-2621.2002.tb10291.x>. Acesso em: 10 jan. 2013. doi: 10.1111/j.1365-2621.2002.tb10291.x.

AOAC (ASSOCIATION OF OFFICIAL ANALYTICAL CHEMISTS). Official methods of analysis. 17.ed. Gaithersburg, MD, 2002. 1170p.

BOURNE, M.C. Texture profile analysis. Food Technology, v.32, n.7, p.62-72, 1978.

BRASIL. Ministério da Agricultura, Pecuária e Abastecimento (MAPA). Secretaria de Defesa Agropecuária (SDA). Instrução normativa n.4, 31 março de 2000. Aprova os Regulamentos Técnicos de Identidade e Qualidade de Carne Mecanicamente Separada, de Mortadela, de Lingüiça e de Salsicha. Diário Oficial da União, Brasília, seção 1, p.6-10, 2000.

BREWER, M.S. Irradiation effects on meat flavor: a review. Meat Science, v.81, p.1-14, 2009. Disponível em: <http://dx.doi. org/10.1016/j.meatsci.2008.07.011>. Acesso em: 21 jan. 2013. doi: 10.1016/j.meatsci.2008.07.011.

BYUN, M.W. et al. Effect of gamma irradiation on the raw meat in Bologna sausage production. International Journal of Food Science and Technology, v.35, n.6, p.599-601, 2000. Disponível em: <http://dx.doi.org/10.1111/j.1365-2621.2000.00409.x>. Acesso em: 10 fev. 2013. doi: 10.1111/j.1365-2621.2000.00409.x.

CABEZA, M.C. et al. Safety and quality of ready-to-eat dry fermented sausages subjected to E-beam radiation. Meat Science, v.83, p.320-327, 2009. Disponível em: <http://dx.doi. org/10.1016/j.meatsci.2009.05.019>. Acesso em: 16 dez. 2012. doi: 10.1016/j.meatsci.2009.05.019.

CAMMACK, R. et al. Nitrite and nitrosyl compounds in food preservation. Biochimica et Biophysica Acta - Bioenergetics, 
v.1411, n.2-3, p.475-488, 1999. Disponível em: <http://dx.doi. org/10.1016/S0005-2728(99)00033-X>. Acesso em: 8 jan. 2013 doi: 10.1016/S0005-2728(99)00033-X.

DONG, Q.L. et al. The effect of sodium nitrite on the textural properties of cooked sausage during cold storage. Journal of Texture Studies, v.38, p.537-554, 2007. Disponível em: <http:// dx.doi.org/10.1111/j.1745-4603.2007.00112.x>. Acesso em: 8 jan. 2013. doi: 10.1111/j.1745-4603.2007.00112.x.

DUTRA, M.P. et al. Radiação gama e tempo de armazenamento sobre a oxidação lipídica, cor objetiva, pigmentos heme e nitrito residual de mortadelas elaboradas com diferentes níveis de nitrito. Ciência Rural, v.41, n.12, p.2203-2209, 2011. Disponível em: <http://dx.doi.org/10.1590/S0103-84782011005000143>. Acesso em: 30 jan. 2013. doi: 10.1590/S0103-84782011005000143.

GALÁN, I. et al. Effects of ionising irradiation on quality and sensory attributes ofready-to-eat dry fermented sausages enriched with folic acid. International Journal of Food Science and Technology, v.46, p.469-477, 2011. Disponível em: <http://dx.doi. org/10.1111/j.1365-2621.2010.02534.x>. Acesso em: 2 fev. 2013. doi: 10.1111/j.1365-2621.2010.02534.x.

HONIKEL, K.O. The use and control of nitrate and nitrite for the processing of meat products. Meat Science, v.78, p.68-76, 2008 Disponível em: <http://dx.doi.org/10.1016/j.meatsci.2007.05.030>. Acesso em: 15 fev. 2013. doi: 10.1016/j.meatsci.2007.05.030.

HOUSER, T.A. et al. Effects of irradiation on properties of cured ham. Journal of Food Science, v.68, p.2362-2365, 2003.
Disponível em: <http://onlinelibrary.wiley.com/doi/ 10.1111/ j.1365-2621.2003.tb05773.x/pdf>. Acesso em: 20 nov. 2013. doi: 10.1111/j.1365-2621.2003.tb05773.x.

MOHLER, K.; SCHEERER, C. Bilanz der bildung von pokelfarbstoff im muskelfleisch. v. reaktionen von nitrit mit schweinemuskel. Zeitschrift fur Lebensmittel-Untersuchung und -Forschung, v.168, n.3, p.173-176, 1979.

PEREIRA, A.G.T. et al. Effects of the addition of mechanically deboned poultry meat nd collagen fibers on quality characteristics of frankfurter-type sausages. Meat Science, v.89, n.4, p.519-525, 2011. Dispoinível em: <http://www.sciencedirect.com/science/ article/pii/S0309174011002099>. Acesso em: 18 nov. 2013. doi: 10.1016/j.meatsci.2011.05.022.

RAMOS, E.M.; GOMIDE, L.A.M. Avaliação da qualidade de carnes: fundamentos e metodologias. Viçosa: UFV, 2007. 599p.

SOKHEY, A.S.; HANNA, M.A. Properties of irradiated starches. Food Structure, v.12, n.397-410, 1993.

STEWART, E.M. Food irradiation chemistry. In: MOLINS, R.A. (Ed.). Food irradiation: principles and applications. New York: John Wiley \& Sons, 2001. p.37-76.

THAKUR, B.R.; SINGH, R.K. Food irradiation: chemistry and applications. Food Reviews International, v.10, n.4, p.437-473, 1994. Disponível em: <http://dx.doi.org/10.1 080/87559129409541012>. Acesso em: 4 fev. 2013. doi: $10.1080 / 87559129409541012$. 